\title{
Status and outlook of the neutron time-of-flight facility n_TOF at CERN
}

F. Gunsing ${ }^{\mathrm{a}, *}$, U. Abbondanno ${ }^{\mathrm{b}}$, G. Aerts ${ }^{\mathrm{a}}, \mathrm{H}$. Álvarez ${ }^{\mathrm{c}}$, F. Álvarez-Velarde ${ }^{\mathrm{d}}$, S. Andriamonje a , J. Andrzejewski e, P. Assimakopoulos ${ }^{\text {f }}$, L. Audouin ${ }^{\mathrm{g}}$, G. Badurek h, P. Baumann i , F. Bečváŕ ${ }^{j}$, E. Berthoumieux ${ }^{a}, F$. Calviño ${ }^{k}$, D. Cano-Ott ${ }^{d}$, R. Capote ${ }^{1, m}$, A. Carrillo de Albornoz ${ }^{n}$, P. Cennini ${ }^{\circ}$, V. Chepel ${ }^{p}$, E. Chiaveri ${ }^{o}$, N. Colonna ${ }^{\mathrm{q}}$, G. Cortes ${ }^{\mathrm{r}}$, A. Couture ${ }^{\mathrm{s}}, \mathrm{J}$. Cox ${ }^{\mathrm{s}}$, M. Dahlfors ${ }^{\mathrm{o}}$, S. David ${ }^{\mathrm{g}}$, I. Dillman ${ }^{\mathrm{t}}$, R. Dolfini ${ }^{\mathrm{u}}$, C. Domingo-Pardo v, W. Dridi ${ }^{\mathrm{a}}$, I. Duran ${ }^{\text {c }}$, C. Eleftheriadis ${ }^{\text {w }}$, L. Ferrant ${ }^{\mathrm{g}}$, A. Ferrari ${ }^{\text {o }}$, R. Ferreira-Marques ${ }^{p}$, H. Frais-Koelbl ${ }^{1}$, K. Fujii ${ }^{b}$, W. Furman ${ }^{x}$, I. Goncalves ${ }^{p}$,

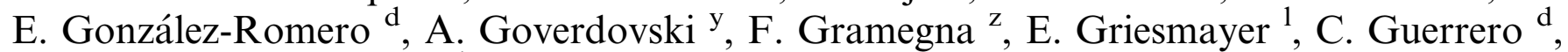
B. Haas ${ }^{\text {aa }}$, R. Haight ${ }^{\text {ab }}$, M. Heil ${ }^{\mathrm{t}}$, A. Herrera-Martinez ${ }^{\mathrm{o}}$, M. Igashira ${ }^{\text {ac }}$, S. Isaev ${ }^{\mathrm{g}}$, E. Jericha h ${ }^{\text {, F. Käppeler }}{ }^{\text {t, Y. Kadi }}{ }^{o}$, D. Karadimos ${ }^{f}$, D. Karamanis ${ }^{f}$, M. Kerveno ${ }^{i}$, V. Ketlerov ${ }^{y, o}$, P. Koehler ad, V. Konovalov ${ }^{x, o}$, E. Kossionides ${ }^{\text {ae }}$, M. Krtička ${ }^{j}$, C. Lampoudis ${ }^{\text {w,a }}$, H. Leeb ${ }^{\mathrm{h}}$, A. Lindote ${ }^{\mathrm{p}}$, I. Lopes ${ }^{\mathrm{p}}$, M. Lozano ${ }^{\mathrm{m}}$, S. Lukic ${ }^{\mathrm{i}}$, J. Marganiec e , L. Marques ${ }^{\text {n, S. Marrone }}{ }^{\mathrm{q}}$, T. Martínez $^{\mathrm{d}}$, C. Massimi af ${ }^{\text {, P. Mastinu }}{ }^{\mathrm{z}}$, A. Mengoni ${ }^{1, o}$, P.M. Milazzo ${ }^{\text {b }}$, C. Moreau ${ }^{b}$, M. Mosconi ${ }^{t}$, F. Neves ${ }^{p}$, H. Oberhummer ${ }^{\text {h }}$, S. O'Brien ${ }^{\mathrm{s}}$, M. Oshima ${ }^{\mathrm{ag}}$, J. Pancin ${ }^{\mathrm{a}}$, C. Papachristodoulou ${ }^{\mathrm{f}}, \mathrm{C}$. Papadopoulos ${ }^{\text {ah }}$, C. Paradela ${ }^{c}$, N. Patronis ${ }^{\mathrm{f}}$, A. Pavlik ${ }^{\text {ai }}$, P. Pavlopoulos ${ }^{\text {aj }}$, L. Perrot ${ }^{\text {a }}$, M.T. Pigni ${ }^{\text {h }}$, R. Plag ${ }^{\mathrm{t}}$, A. Plompen ${ }^{\mathrm{ak}}$, A. Plukis ${ }^{\mathrm{a}}$, A. Poch ${ }^{\mathrm{r}}$, C. Pretel ${ }^{\mathrm{r}}$, J. Quesada ${ }^{\mathrm{m}}$, T. Rauscher ${ }^{\mathrm{al}}$, R. Reifarth ${ }^{\mathrm{ab}}$, M. Rosetti ${ }^{\text {am }}$, C. Rubbia ${ }^{\text {u }}$, G. Rudolf ${ }^{\mathrm{i}}$, P. Rullhusen ${ }^{\mathrm{ak}}$, J. Salgado ${ }^{\mathrm{n}}$, L. Sarchiapone ${ }^{\text {o, I. Savvidis }}{ }^{\text {w }}$, C. Stephan ${ }^{\mathrm{g}}$, G. Tagliente ${ }^{\mathrm{q}}$, J.L. Tain ${ }^{\mathrm{v}}$, L. Tassan-Got ${ }^{\mathrm{g}}$, L. Tavora ${ }^{\mathrm{n}}$, R. Terlizzi ${ }^{\mathrm{q}}$, G. Vannini ${ }^{\text {af }}$, P. Vaz $^{\mathrm{n}}$, A. Ventura ${ }^{\mathrm{am}}$, D. Villamarin ${ }^{\mathrm{d}}$, M.C. Vincente ${ }^{\mathrm{d}}$, V. Vlachoudis ${ }^{\circ}$, R. Vlastou ${ }^{\text {ah }}$, F. Voss ${ }^{\mathrm{t}}$, S. Walter ${ }^{\mathrm{t}}$, H. Wendler ${ }^{\circ}$, M. Wiescher ${ }^{\mathrm{s}}$, K. Wisshak ${ }^{\mathrm{t}}$, the $\mathrm{n} \_\mathrm{TOF}$ Collaboration

\footnotetext{
${ }^{a}$ CEA/Saclay - DSMIDAPNIA, Gif-sur-Yvette, France

${ }^{\mathrm{b}}$ Istituto Nazionale di Fisica Nucleare, Trieste, Italy

${ }^{\mathrm{c}}$ Universidade de Santiago de Compostela, Spain

${ }^{\mathrm{d}}$ Centro de Investigaciones Energeticas Medioambientales y Technologicas, Madrid, Spain ${ }^{\mathrm{e}}$ University of Lodz, Lodz, Poland

${ }^{\mathrm{f}}$ University of Ioannina, Greece

${ }^{g}$ Centre National de la Recherche Scientifique/IN2P3 - IPN, Orsay, France

${ }^{\mathrm{h}}$ Atominstitut der Österreichischen Universitäten, Technische Universität Wien, Austria

${ }^{i}$ Centre National de la Recherche ScientifiquelIN2P3 - IReS, Strasbourg, France

${ }^{\mathrm{j}}$ Charles University, Prague, Czech Republic

${ }^{\mathrm{k}}$ Universidad Politecnica de Madrid, Spain

${ }^{1}$ International Atomic Energy Agency (IAEA), Nuclear Data Section, Vienna, Austria

${ }^{\mathrm{m}}$ Universidad de Sevilla, Spain

${ }^{\mathrm{n}}$ Instituto Tecnológico e Nuclear(ITN), Lisbon, Portugal

${ }^{\circ}$ CERN, Geneva, Switzerland

${ }^{\mathrm{p}}$ LIP - Coimbra \& Departamento de Fisica da Universidade de Coimbra, Portugal

${ }^{\mathrm{q}}$ Istituto Nazionale di Fisica Nucleare, Bari, Italy
} 


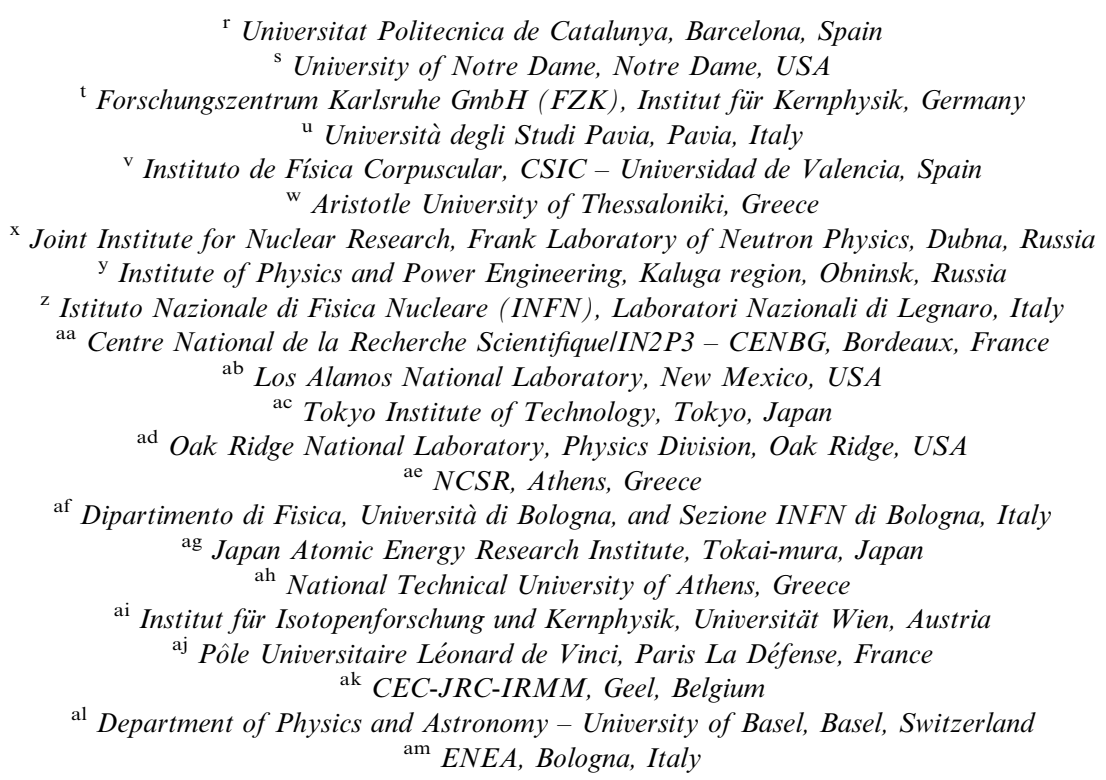

Available online 25 May 2007

\begin{abstract}
The neutron time-of-flight facility n_TOF at CERN, fully operational since 2002, combines a high instantaneous neutron flux with high energy resolution. The wide energy range and the high neutron flux per time-of-flight burst result in a much enhanced signal to background ratio for neutron capture of radioactive isotopes and makes this facility well suited for the measurement of high quality neutron-induced reaction cross-sections.

Neutrons are created by spallation reactions induced by a pulsed $20 \mathrm{GeV} / \mathrm{c}$ proton beam impinging on a lead target. A $5 \mathrm{~cm}$ water slab surrounding the lead target serves as a coolant and at the same time as a moderator of the spallation neutron spectrum, providing a wide energy spectrum from $0.1 \mathrm{eV}$ to about $250 \mathrm{MeV}$.

By the end of 2005, a first phase of data taking has been successfully terminated. Fission and capture experiments have been performed on a variety of isotopes of interest for nuclear astrophysics, advanced nuclear technologies and for basic nuclear physics.

The instrumentation developed for this facility consists of parallel plate avalanche counter and fission ionization chamber detectors for the fission experiments and of low mass $\mathrm{C}_{6} \mathrm{D}_{6}$ detectors and a 4 pi $\mathrm{BaF}_{2}$ total absorption calorimeter for capture experiments. A new data acquisition system, based on sampling of the detector signals, has been developed to cope with the high count rates and to minimize the effective dead time to only a few tens of nanosecond.

A second phase of data taking is planned to start in 2007, after an upgrade of the spallation target. On the longer term, the construction of a flight path at $20 \mathrm{~m}$ resulting in an increased neutron flux of about a factor of 100 opens new possibilities.
\end{abstract}

(C) 2007 Elsevier B.V. All rights reserved.

PACS: $29.25 ; 28.20 .-\mathrm{v} ; 24.30 .-\mathrm{v} ; 98.80$

Keywords: Neutrons; Time-of-flight; CERN; Spallation; n_TOF

\section{Introduction}

The detailed study of neutron-nucleus reactions is of main importance for a wide variety of research fields ranging from stellar nucleosynthesis [1,2], neutron-induced symmetry breaking $[3,4]$ and nuclear level density investigation [5-7] to applications of nuclear technology [8-10] like the transmutation of nuclear waste, accelerator driven

\footnotetext{
* Corresponding author. Tel.: +331690875 23; fax: +33169087584. E-mail address: gunsing@cea.fr (F. Gunsing).
}

systems (ADS) and the thorium-based nuclear fuel cycle. All these applications have pointed out that for many nuclides the available nuclear data is of insufficient accuracy and sometimes even lacking. Neutron cross-section data are available by means of compilations of experimental data $[11,12]$ or through evaluated nuclear data libraries such as BROND, ENDF, JEFF and JENDL [13]. The necessary update of these neutron cross-section libraries rely on the availability of accurate measurements obtainable at advanced neutron sources.

The new neutron time-of-flight facility $n \_$TOF has been recently constructed at CERN in Geneva for the study of 
neutron-induced reactions for nuclear technology, nuclear astrophysics and nuclear structure and reaction physics. Up to now, the facility has been mainly used to measure neutron capture and fission cross-sections of a large number of isotopes.

\section{The neutron time-of-flight facility n_TOF}

The idea to construct a new neutron time-of-flight facility at CERN has been proposed by Rubbia et al. [14]. The facility has become fully operational with the start of the scientific measurement programme in May 2002. Neutrons are produced by spallation reactions induced by a pulsed, $6 \mathrm{~ns}$ wide, $20 \mathrm{GeV} / \mathrm{c}$ proton beam with up to $7 \times 10^{12}$ protons per pulse, impinging on a $80 \times 80 \times 60 \mathrm{~cm}^{3}$ lead target, yielding about 300 neutrons per incident proton. A $5 \mathrm{~cm}$ water slab surrounding the lead target serves as a coolant and at the same time as a moderator of the initially fast neutron spectrum, providing a wide energy spectrum from $1 \mathrm{eV}$ to about $250 \mathrm{MeV}$ with a nearly $1 / \mathrm{E}$ isolethargic flux dependence up to $1 \mathrm{MeV}$. A vacuum neutron beam line leads to the experimental area at $185 \mathrm{~m}$ from the lead target. A short summary of the full description of the characteristics and performances of the facility described in [15], will be given here.

The neutron beam is obtained by means of two collimators, consisting of layers of iron and borated polyethylene. The iron serves to stop fast neutrons, while the hydrogen in polyethylene moderates the neutrons and boron-10 captures the slower neutrons. One collimator has an inner diameter of $11 \mathrm{~cm}$, an outer diameter of $50 \mathrm{~cm}$ and is placed at $135 \mathrm{~m}$ from the lead target. The second collimator is located near the experimental area at a distance of $175 \mathrm{~m}$ and has an outer diameter of $40 \mathrm{~cm}$ a variable inner diameter. Typically an inner diameter of $1.8 \mathrm{~cm}$ is used for capture measurements and $8 \mathrm{~cm}$ for fission experiments. This collimation results in a nearly symmetric Gaussianshaped beam profile at the sample position with a standard deviation of about $0.77 \mathrm{~cm}$ at low neutron energies with the $1.8 \mathrm{~cm}$ diameter collimator. The spatial distribution has been accurately measured as a function of neutron energy [16].

In order to remove residual charged particles going along the neutron beam line a $1.5 \mathrm{~T}$ sweeping magnet has been placed at a distance of $145 \mathrm{~m}$ of the spallation target. A previously observed background presumably due to negative muon capture has been drastically reduced by means of a $3 \mathrm{~m}$ thick iron shielding located just after the sweeping magnet $[17,18]$. The experimental area where the samples and detectors are mounted for capture and fission experiments ranges from 182 to $190 \mathrm{~m}$. It will be described in more detail in the next section. The neutron beam line is extended for an additional $12 \mathrm{~m}$ beyond the experimental area to minimize the background from back-scattered neutrons.

The neutron kinetic energy is determined by time-offlight. This is possible since the proton beam is pulsed.
The impact of the proton beam on the lead target is closely related to the creation time of neutrons of all energies. The detection of a reaction product of a neutron-induced reaction in the experimental area provides the time the neutron has taken to travel the distance from the spallation target to the experimental area. The velocity and hence the kinetic energy of the neutron can be deduced from the flight time and the flight distance. The repetition period of the proton pulses is at least $2.4 \mathrm{~s}$, which is low enough to cover the energy range down to subthermal energies and to prevent overlapping of slow neutrons in subsequent cycles.

\section{Beam characteristics}

A pulsed white neutron source can be characterized by the energy distribution of its neutron flux, both time-averaged and per time-of-flight bunch and by its energy resolution. The energy distribution of the neutron flux at the sample position at $185 \mathrm{~m}$ for a standard time-of-flight bunch of $7 \times 10^{12}$ protons is shown in Fig. 1. The data are based on a measurement performed with a ${ }^{235} \mathrm{U}$ loaded parallel plate fission ionization chamber from the Physikalisch-Technische Bundesanstalt in Braunschweig [19] and below $1 \mathrm{keV}$ from a ${ }^{6} \mathrm{Li}$-based in-beam neutron flux monitor [20]. The ${ }^{6} \mathrm{Li}$-based flux detector is always present during the measurements. The flux per bunch governs the signal to noise ratio. In capture experiments a high flux per bunch results in a favorable ratio of gamma-rays from $(\mathrm{n}, \gamma)$ reactions to gamma-rays from radioactivity. In order to obtain the average flux, which determines the duration of the experiment, one just multiplies the instantaneous flux with the repetition rate of the proton beam.

A gross knowledge of the energy resolution is sufficient when addressing smooth cross-sections at higher energies. At lower energies in the resolved resonance region however, where the cross-section can vary several orders of magnitude over a few electronvolt, the full distribution of the energy resolution, which is often non-Gaussian, is needed to determine accurately the description of the resonances. The spread in energy for a measured flight time can

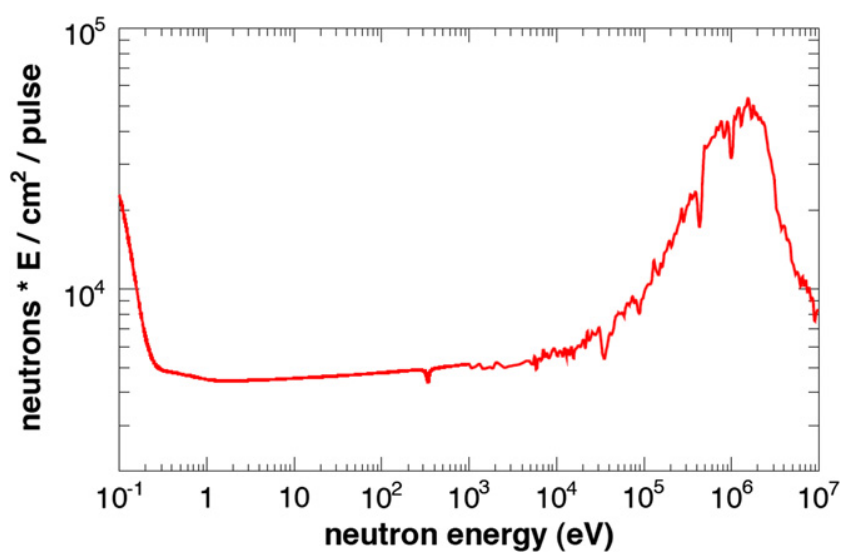

Fig. 1. The number of neutrons per $\mathrm{cm}^{2}$ at the sample position in the experimental area at $185 \mathrm{~m}$ for a standard pulse of $7 \times 10^{12}$ protons. 


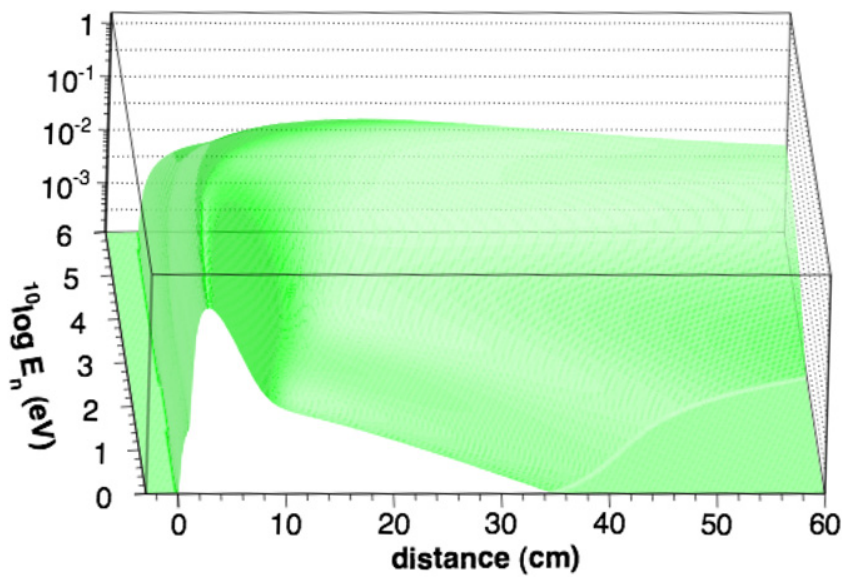

Fig. 2. The resolution function of the $n \_$TOF facility, given as the distribution of the equivalent distance as a function of the neutron energy in the range from $1 \mathrm{eV}$ to $1 \mathrm{MeV}$.

conveniently be expressed in terms of a spread in the effective flight distance. The distribution of the equivalent distance is an appropriate quantity to evaluate the resolution as a function of neutron energy. We obtained the resolution function of the $n \_$TOF facility by two independent simulation codes $[21,22]$ giving consistent results. The Monte Carlo results were then fitted by an analytical two-dimensional function and implemented in the resonance shape analysis code SAMMY [23]. In Fig. 2 the resolution function in the form of the equivalent distance distribution is shown in the relevant energy range from $1 \mathrm{eV}$ to $1 \mathrm{MeV}$ on a logarithmic scale.

\section{Instrumentation}

For the capture and fission experiments several detectors are available. We have used in-house developed deuterated benzene $\mathrm{C}_{6} \mathrm{D}_{6}$ gamma-ray detectors contained in a cylindrical low mass carbon fibre housing [24] for neutron capture measurements on several isotopes. The samples are kept in position by a carbon fiber sample changer. The low neutron capture cross-section of both carbon and deuterium assure a low contribution from sample scattered neutrons to the background. Measurements on the isotopes ${ }^{56} \mathrm{Fe}$, ${ }^{197} \mathrm{Au},{ }^{151} \mathrm{Sm},{ }^{209} \mathrm{Bi}, \quad{ }^{204,206,207,208} \mathrm{~Pb},{ }^{232} \mathrm{Th},{ }^{139} \mathrm{La}$, ${ }^{24,25,26} \mathrm{Mg},{ }^{186,187,188} \mathrm{Os},{ }^{90,91,92,93,94,96} \mathrm{Zr}$ have been performed. Many of these measurements are still under analysis, while for some of them final data have been published [25-30].

A second capture detector has become available in 2004. It is a $4 \pi 100 \%$ efficiency total absorption capture detector made of $42 \mathrm{BaF}_{2}$ crystals in ${ }^{10} \mathrm{~B}$ loaded carbon fibre capsules, coupled to XP4512B photomultipliers. Samples are placed in the center of the calorimeter and are surrounded by a $\mathrm{C}_{12} \mathrm{H}_{20} \mathrm{O}_{4}\left({ }^{6} \mathrm{Li}\right)_{2}$ neutron absorber. Measurements of ${ }^{197} \mathrm{Au},{ }^{234} \mathrm{U},{ }^{237} \mathrm{~Np},{ }^{240} \mathrm{Pu},{ }^{243} \mathrm{Am},{ }^{233} \mathrm{U}$ have been performed. The analysis procedure is rather complex and although intermediate results have been reported already in several conference proceedings, no final data have been published yet.

Fission experiments have been performed with two different detector systems. A fission ionization chamber (FIC) has been developed for use at the n_TOF facility using deposits of fissile isotopes on $100 \mu \mathrm{m}$ thick aluminum foils. Also parallel plate avalanche counters (PPACs) have been developed with target deposits on $1.5 \mu \mathrm{m}$ thin mylar or $2 \mu \mathrm{m}$ aluminum foils, allowing to detect the two fission fragments in coincidence. Data sets have been taken for the isotopes ${ }^{209} \mathrm{Bi}$, ${ }^{\text {nat }} \mathrm{Pb},{ }^{232} \mathrm{Th},{ }^{233,234,235,236,238} \mathrm{U},{ }^{237} \mathrm{~Np}$, ${ }^{241,243} \mathrm{Am}$ and ${ }^{245} \mathrm{Cm}$. The use of standard cross-sections to measure the neutron flux allows also to determine accurate corrections for the fission efficiency. The data is currently still under analysis.

The data acquisition system [31] is based on the sampling of the detector signals in order to extract the deposited energy and the time-of-flight. An array of 54 Acqiris flash ADCs with 8 bit resolution and $1 \mathrm{~ns}$ sampling time with 8 Mbytes of memory was used to record the full detector signal following the start time given by the incident protons. The digitizers were operated at $500 \mathrm{M}$ samples/s allowing to store the detector signal during $16 \mathrm{~ms}$, corresponding to a time-of-flight of $0.3 \mathrm{eV}$. After zero suppression, the data are sent to CERN's data storage facility CASTOR for off-line analysis with dedicated pulse shape analysis routines for each detector.

\section{Outlook}

After the successful completion of data taking during the first phase of data taking of the $n \_$TOF facility from 2002 to 2004, an upgrade of the spallation target is foreseen for 2007 and a new series of capture and fission measurements is planned for a second phase of operation. On the longer term, we plan to construct a second flight path at the shorter distance of $20 \mathrm{~m}$. The increased flux of about a factor of hundred opens a wide range of new possibilities for measurements with even lower mass samples.

\section{References}

[1] G. Wallerstein et al., Rev. Mod. Phys. 69 (1997) 995.

[2] F. Käppeler, Progr. Particle Nucl. Phys. 43 (1999) 419.

[3] G.E. Mitchell, J.D. Bowman, H.A. Weidenmüller, Rev. Mod. Phys. 71 (1999) 445.

[4] Y. Masuda et al., Nucl. Phys. A 721 (2003) 485C.

[5] T. von Egidy, D. Bucurescu, Phys. Rev. C 72 (2005) 044311.

[6] H. Nakamura, T. Fukahori, Phys. Rev. C 72 (2005) 064329.

[7] W.P. Abfalterer, R.W. Finlay, S.M. Grimes, Phys. Rev. C 72 (2000) 064312

[8] M. Salvatores, I. Slessarev, A. Tchistiakov, Nucl. Sci. Eng. 130 (1998) 309.

[9] W. Gudowski, Nucl. Phys. A 654 (1999) 436c.

[10] S. David et al., Nucl. Instr. and Meth. A 443 (2000) 510.

[11] S.F. Mughabghab, Atlas of Neutron Resonances, Elsevier, 2005.

[12] S.I. Sukhoruchkin, Z.N. Soroko, V.V. Deriglazov, Low Energy Neutron Physics, Tables of Neutron Resonance Parameters, Vol. I/ 16B, Springer, Landolt-Börnstein, 1998. 
[13] See for example http://www.nndc.bnl.gov, http://www-nds.iaea.org, or http://www.nea.fr.

[14] C. Rubbia et al., Tech. Rep. CERN/LHC/98-02, CERN, 1998.

[15] U. Abbondanno et al., Tech. Rep. CERN-SL-2002-053 ECT, 2003.; C. Borcea et al., Nucl. Instr. and Meth. A 513 (2003) 524.

[16] J. Pancin et al., Nucl. Instr. and Meth. A 524 (2004) 102.

[17] U. Abbondanno et al., Tech. Rep. CERN/INTC 2001-038, 2001.

[18] A. Ferrari, C. Rubbia, V. Vlachoudis, Tech. Rep. CERN-SL-EET2001-036, CERN, 2002.

[19] C. Borcea et al., Nucl. Instr. and Meth. A 513 (2003) 524.

[20] S. Marrone et al., Nucl. Instr. and Meth. A 517 (2004) 389.

[21] C. Coceva, M. Frisoni, M. Magnani, A. Mengoni, Nucl. Instr. and Meth. A 489 (2002) 346.
[22] V. Vlachoudis et al., private communication.

[23] N.M. Larson, SAMMY, Computer code, Report ORNL/TM-9179/ R7, Oak Ridge National Laboratory, 2006.

[24] R. Plag et al., Nucl. Instr. and Meth. A 496 (2003) 425.

[25] U. Abbondanno et al., Nucl. Instr. and Meth. A 521 (2004) 454.

[26] G. Lorusso et al., Nucl. Instr. and Meth. A 532 (2004) 622.

[27] U. Abbondanno et al., Phys. Rev. Lett. 93 (2004) 161103.

[28] S. Marrone et al., Phys. Rev. C 73 (2006) 03604.

[29] G. Aerts et al., Phys. Rev. C 73 (2006) 054610.

[30] C. Domingo-Pardo et al., Phys. Rev. C 74 (2006) 025807.

[31] U. Abbondanno et al., Nucl. Instr. and Meth. A 538 (2005) 692. 